\section{Racial and Ethnic Disparities in Pediatric Kidney Transplantation - Has KAS made a difference?}

Olga Charnaya ${ }^{1}, \mathrm{MD}, \mathrm{MS}$, Sile Yu², MS, Aviva Goldberg 3 , MD, MA, Jacqueline Garonzik-Wang ${ }^{1}, M D, P h D$, Dorry Segev ${ }^{1}, M D, P h D$ and Priya S. Verghese ${ }^{4}$, MD, MPH

${ }^{1}$ Johns Hopkins University, School of Medicine, ${ }^{2}$ Johns Hopkins University, ${ }^{3}$ University of Manitoba and ${ }^{4}$ Northwestern University Feinberg School of Medicine

ABSTRACT IMPACT: Evaluate the impact that the Kidney Allocation System has had on racial and ethnic disparities in pediatric deceased donor kidney transplant recipients. OBJECTIVES/GOALS: Racial and ethnic minority pediatric transplant candidates have known disparities in access to kidney transplantation. The Kidney Allocation System (KAS), implemented in 2014, was designed in part to alleviate some of these disparities thereby making transplant more equitable. We investigated the effect of KAS on reported disparities. METHODS/STUDY POPULATION: We utilized Scientific Registry of Transplant Recipients (SRTR) data to determine differences in new waitlist registrants, deceased donor (DDKT) and living donor kidney transplants (LDKT), HLA mismatch, and allograft survival among pediatric patients of different racial and ethnic backgrounds. RESULTS/ANTICIPATED RESULTS: Black pediatric patients represented $21.3 \%$ of new waitlist registrants pre-KAS and $18.9 \%$ post-KAS. Waitlist time increased for pediatric patients of all races post-KAS with the highest increase (131 days) in Asian patients $(\mathrm{p}<0.01)$. The racial distribution of DDKT pre- and post-KAS was unchanged (White $38.4 \%$ vs $38.3 \%$, Black $24.5 \%$ vs $22.5 \%$, Hispanic $30.6 \%$ vs $31.1 \%$, Asian $3.7 \%$ vs $4.4 \%$, p = 0.12 ). The 3 -yrgraft failure rate is disproportionately worse in Black children compared to other races pre- and post-KAS (White $6.8 \%$ vs $5.3 \%$, Black $14 \%$ vs $8.7 \%$, Hispanic $8 \%$ vs $4.5 \%$, Asian $6.6 \%$ vs $6.7 \%$, Other $6.5 \%$ vs $2.9 \%$ ) although there is a trend towards better graft survival in the post-KAS era. Graft survival worsened in Asian children in the post-KAS era (HR 2.34,95\% CI 1.05 - 5.25, p=0.038). DISCUSSION/SIGNIFICANCE OF FINDINGS: Racial and ethnic disparities in pediatric ESRD patients have not been ameliorated by KAS. Children of color have longer waitlist time and are more likely to have graft failure. Alarmingly, allograft failure rate increased in Asian patients post-KAS, which merits further evaluation.

\section{Data Science/Biostatistics/Informatics}

\section{9}

Developing the COmmuNity kNowlEdge to aCtion Toolkit (CONNECT) to reduce breast cancer screening disparities Let's CONNECT

David Haynes ${ }^{1}$ and Kelly Hughes ${ }^{2}$

${ }^{1}$ University of Minnesota Institute for Health Informatics and

${ }^{2}$ Minnesota Department of Health

ABSTRACT IMPACT: Advanced spatial analysis techniques are used to target a community education intervention for Immigrant and African American women to increase breast cancer screening. OBJECTIVES/GOALS: We are addressing breast cancer screening disparities through the development of the COmmuNity kNowlEdge to aCtion Toolkit (CONNECT). CONNECT implements a mixed-methods approach using GIScience, community education, and social media to mitigate the impact of breast cancer screening disparities for Immigrant African and African American women. METHODS/STUDY POPULATION: We used advanced spatial analysis techniques, Spatially Adaptive Filters (SAF) to reveal mammography screening rates below the state level. SAF create screening maps of This new information allows lay health educators to identify and engage their communities in the Breast Cancer Champions program. We transformed and curated existing cancer educational material into culturally relevant educational training for lay health educators. Lay health educators participate in educational trainings and receive stipends for conducting formal and informal breast cancer education and screening events. RESULTS/ ANTICIPATED RESULTS: We have identified four principles for designing culturally relevant education materials.

1. Visual representation of the community in materials

2. Positive Framing

3. Statistics and graphs should be minimal

4. Appropriate reading level and minimizing jargon

Due to COVID-19, our breast cancer champions are engaging with their community in socially responsible ways (i.e., engaging through social media, developing and placing community education flyers, community radio spots). Our social media campaign, which began in October has already attracted over 1000 followers. DISCUSSION/SIGNIFICANCE OF FINDINGS: Despite the disruption of COVID-19, our project continues reduce breast cancer screening disparities. We have developed and created culturally appropriate materials and are currently training Champions. By incorporating an online presence into our community outreach, we are increasing the ways we connect with our community.

\section{Digital Health/Social Media}

\section{8}

\section{A Mixed Method Study: Can Lyft Facilitate Better Access to Healthy Food?* \\ Raneitra Grover and Lenwood Hayman \\ Morgan State University School of Community Health and Policy}

ABSTRACT IMPACT: Interventions designed to improve access to healthy food are needed as a mechanism to improve diet quality and ultimately prevent diet-related chronic diseases. OBJECTIVES/ GOALS: For people living in food deserts, access to grocery stores is an important consideration toward improving diet quality. The Grocery Access Program (GAP) provided discounted Lyft rides to grocery stores for residents in Baltimore, MD. This study will assess how the GAP impacted access to different food retail stores and healthy food purchases. METHODS/STUDY POPULATION: A mixed methods sequential explanatory design will be used. We collected survey data at baseline and mid-pilot on primary grocery shopping store, frequency of purchasing fruits and vegetables, and frequency of using the discounted Lyft rides for 90 program enrollees. The Healthy Food Availability Index (HFAI), a validated, 